\title{
Verbleib der Geraer Silbermöbel im Verzeichnis national wertvollen Kulturgutes
}

Thüringer Oberverwaltungsgericht, Beschluss vom 22. November 2007 - 1 ZKO 1000/06

\section{Die Eintragung eines Kulturgutes in das „Verzeichnis national wertvollen Kulturgutes“ (§ 1 KultgSchG) ist ein Verwaltungsakt. Dieser wird nach Maßgabe des § 6 Abs. 1 Satz 1 KultgSchG öffentlich bekannt gemacht.}

2. Soweit im Kulturgutschutzgesetz von einer „Entscheidung“ ̈uber die Eintragung die Rede ist, handelt es sich um das der Eintragung vorgelagerte Verfahren der Entscheidungsfindung, das noch nicht auf Außenwirkung gerichtet ist.

3. Auch für Kulturgüter, die bereits unter dem Schutz der „Verordnung über die Ausfuhr von Kunstwerken“ vom 11.12.1919 standen, ist ein Neueintragungsverfahren nach Maßgabe des § 1 KultgSchG vorgesehen. $\$ 22$ Abs. 3 KultgSchG ordnet an, dass die Ausfuhr dieser Kulturgüter schon vor einer Eintragung erforderlich ist. Eine Regelung über ein gesondertes Übernahmeverfahren für diese Kulturgüter enthält § 22 KultgSchG nicht.

\section{$[\ldots]$}

Der Antrag auf Zulassung der Berufung gegen das Urteil des Verwaltungsgerichts Gera vom 27. September 2006 - 2 K 382/06 $\mathrm{Ge}$ - wird abgelehnt. Die Klägerin hat die Kosten des Verfahrens zu tragen. Der Streitwert wird für das Zulassungsverfahren auf 2.000.000,00 € festgesetzt.

Gründe:

I.

Die Klägerin begehrt die Löschung einer Eintragung von Silbermöbeln aus dem Gesamtverzeichnis national wertvollen Kulturgutes.

Im 18. Jahrhundert ließ Heinrich XXV. Graf Reuß-Gera in Augsburg die sog. "Geraer Silbermöbel“, bestehend aus einem Paar Barock Gueridons, einem Barocktisch und einem Silberspiegel, herstellen. Die Silbermöbel waren 1938 in dem Verzeichnis der „Verordnung über die Ausfuhr von Kunstwerken“ vom 11. Dezember 1919 eingetragen. Der Silberspiegel ging im Jahre 1945 in den Kriegswirren verloren. Der damalige Eigentümer R1 wurde im Jahre 1945 besatzungshoheitlich enteignet und sein Vermögen in Volkseigentum überführt. Die "Geraer Silbermöbel“ erhielt seinerzeit die Stadt Gera.

Im Oktober 1990 meldete R2 als Rechtsnachfolgerin des R1 Restitutionsansprüche bezogen auf das ihm entzogene Vermögen an. Am 21. Oktober 1997 schlossen die Stadt Gera und R2 einen Vertrag, in dem ihr im Rahmen einer gütlichen Einigung über die Erfüllung von Ansprüchen nach dem Ausgleichsleistungsgesetz u. a. die „Geraer Silbermöbel“ übereignet wurden. Durch Bescheid vom 16. Januar 1998 bestätigte das Thüringer Landesamt zur Regelung offener Vermögensfragen diese gütliche Einigung.

Im November 1997 leitete der Beklagte ein Verfahren zur Eintragung der "Geraer Silbermöbel“ in das „Verzeichnis national wertvollen Kulturgutes" ein. Mit Schreiben vom 15. September 1998 teilte der Beklagte der „Familie R, Büro R3“ mit, dass die
"Geraer Silbermöbel" in das Gesamtverzeichnis national wertvollen Kulturgutes eingetragen werden sollten.

Dieses Schreiben beantwortete die „R GbRmbH - Geschäftsführung Büro R3" mit Schreiben vom 22. September 1998 und bat darum, alle Schreiben an diese Gesellschaft zu richten. Unterschrieben war dieses Schreiben von einem Herrn T.

Am 6. Oktober 1998 machte der Beklagte die Eintragung der "Geraer Silbermöbel“ in das Gesamtverzeichnis national wertvollen Kulturgutes bekannt. Mit Schreiben vom 16. Dezember 1998 setzte der Beklagte die R GbR-mbH von der Eintragung in Kenntnis.

Mit Vertrag vom 15. Juni 2002 übereignete R2 die "Geraer Silbermöbel“ ihrem Sohn R4, der am gleichen Tag die Klägerin gründete und die Silbermöbel in diese Gesellschaft einbrachte. Die Klägerin übereignete die Silbermöbel im Jahre 2003 zur Sicherheit an eine Bank.

Am 31. Mai 2005 beantragte die Klägerin die Löschung der Eintragung der Geraer Silbermöbel aus dem Verzeichnis national wertvollen Kulturgutes. Diesen Antrag lehnte der Beklagte durch Bescheid vom 28. März 2006 ab. Die hiergegen erhobene Klage hat das Verwaltungsgericht Gera durch Urteil vom 27. September 2006 - 2 K 382/06 Ge - abgewiesen: Es könne dahinstehen, ob der Verwaltungsakt zur Eintragung der "Geraer Silbermöbel" in der Entscheidung des Beklagten vom 6. Oktober 1998 oder in dem Schreiben vom 16. Dezember 1998 zu sehen sei, da diese nicht streitgegenständlich seien. Die Entscheidung des Beklagten vom 6. Oktober 1998 sei jedenfalls mit dem Schreiben vom 16. Dezember 1998 bekannt gemacht worden und deshalb wirksam. § 19 des Gesetzes zum Schutz deutschen Kulturgutes gegen Abwanderung - KultgSchG - stehe der Anwendbarkeit dieses Gesetzes nicht entgegen. Unerheblich sei auch, dass die "Geraer Silbermöbel" bereits aufgrund einer Verordnung aus dem Jahre 1919 gegen eine Verbringung ins Ausland geschützt gewesen seien. § 22 KultgSchG regele für diese Kulturgüter nur eine Genehmigungspflicht. Ein Löschungsanspruch nach § 7 KultgSch G bestehe nicht, weil nach der Eintragung 1998 keine wesentliche Änderung der Umstände eingetreten sei. Das sog. „Welfensilber“ 
sei schon im Jahre 1987 vor der Eintragung der Geraer Silbermöbel in das Gesamtverzeichnis national wertvollen Kulturgutes aufgenommen worden. Das von der Klägerin in Abrede gestellte öffentliche Interesse an den Silbermöbeln begründe auch keinen nachträglichen Umstand, da dieses nicht prägend für den Begriff des national wertvollen Kulturgutes sei.

\section{II.}

Der Antrag der Klägerin auf Zulassung der Berufung hat keinen Erfolg. Die geltend gemachten Zulassungsgründe des § $124 \mathrm{Abs}$. 2 Nr. 1, 2 und 3 VwGO liegen nicht vor.

1.) Die Entscheidung des Verwaltungsgerichts begegnet keinen ernstlichen Zweifeln. Ernstliche Zweifel an der Richtigkeit der erstinstanzlichen Entscheidung (§ 124 Abs. 2 Nr. 1 VwGO) sind nur dann anzunehmen, wenn ein einzelner tragender Rechtssatz oder eine erhebliche Tatsachenfeststellung mit schlüssigen Argumenten in Frage gestellt wird (vgl. BVerfG, Beschluss vom 26. März 2007 - 1 BvR 2228/02 - BayVBL. 2007, S. 624). Diesen Anforderungen werden die Darlegungen der Klägerin nicht gerecht.

a. Soweit die Klägerin geltend macht, die Eintragung der "Geraer Silbermöbel" in das "Verzeichnis nationalen Kulturgutes" im Jahre 1998 sei unwirksam, rechtfertigt dieser Vortrag schon allein deshalb nicht die Annahme ernstlicher Zweifel an der Richtigkeit der Entscheidung, weil die seinerzeitige Eintragung wirksam ist. Die Klägerin macht geltend, der Eintragung müsse eine Entscheidung in Form eines vorgeschalteten Verwaltungsaktes vorausgehen, der den Beteiligten gegenüber bekannt zu machen sei. Ein solcher Verwaltungsakt existiere jedoch nicht. Insbesondere gebe es keine Entscheidung des Beklagten vom 6. Oktober 1998. Es sei nur der Vollzug einer Entscheidung, nämlich die Eintragung mit Schreiben vom 16. Dezember 1998 bekannt gegeben worden. Diese Auffassung findet jedoch keine Stütze im Kulturgutschutzgesetz. Die Bestimmungen des Kulturgutschutzgesetzes sind in der Weise auszulegen, dass die Eintragung nicht nur ein Realakt, sondern selbst der maßgebliche Verwaltungsakt ist, der ggfs. mit Rechtsmitteln angefochten werden kann. Das ergibt sich im Wesentlichen daraus, dass erst durch die Eintragung die im Kulturgutschutzgesetz angeordneten Rechtsfolgen wie insbesondere die Genehmigungsbedürftigkeit der Ausfuhr (§ 1 Abs. 4 KultgSchG) eintreten. Erst mit der Eintragung (und ihrer ordnungsgemäßen Bekanntmachung) wird das Eintragungsverfahren abgeschlossen.

Es ist nicht ersichtlich, wofür der Erlass eines der Eintragung vorgelagerten Bescheides Anknüpfungspunkt sein könnte. Insbesondere begründet eine solche der Eintragung vorangehende Entscheidung keine Rechte und Pflichten im Sinne einer Regelung (§ 35 Satz 1 ThürVwVfG). Gegen die Notwendigkeit, vor der Eintragung einen gesonderten Bescheid zu erlassen, spricht auch $\S 4$ KultgSchG, wonach ein gesetzliches Ausfuhrverbot schon ab Einleitung des Eintragungsverfahrens angeordnet wird. Auch dafür ist also nicht der Erlass eines Verwaltungsaktes erforderlich. Für die Einordnung der Eintragung als Verwaltungsakt spricht auch der Umstand, dass die von der Klägerin begehrte Löschung nach $\S 7$ KultgSchG als „, actus contrarius" ausreicht, um die durch die Eintragung begründeten Rechte und Pflichten nach dem Kulturgutschutzgesetz entfallen zu lassen. Dafür, dass ein weiterer Verwaltungsakt widerrufen oder zurückgenommen werden müsste, gibt es keinen Anhaltspunkt.

Entgegen der Auffassung der Klägerin zwingt auch die Verwendung des Wortes "Entscheidung" in den $\S \S 2$ Abs. 1 und 4 KultgSchG nicht zu einer anderen Einschätzung. Soweit in den Bestimmungen des KultgSchG von einer "Entscheidung“ über die Eintragung die Rede ist, beschreibt dies das der Eintragung vorgelagerte - interne - Verfahren der Entscheidungsfindung. Die zuständige Landesbehörde hat, bevor sie eine Eintragung veranlasst, notwendigerweise eine Prüfung vorzunehmen, ob die Eintragungsvoraussetzungen vorliegen oder nicht. Die „Entscheidung" über die Eintragung schließt diese Prüfung ab, die jedem Erlass eines Verwaltungsaktes denklogisch vorausgeht, bleibt aber ein Internum. Erst die Eintragung, die auf unmittelbare Rechtswirkung nach außen gerichtet ist ( $\$ 35$ Satz 1 Thüringer Verwaltungsverfahrensgesetz - ThürVwVfG -), hat Regelungscharakter. Die Eintragung der "Geraer Silbermöbel" wurde auch ordnungsgemäß öffentlich bekannt gemacht, denn sie wurde gemäß § 6 Abs. 1 Satz 1 KultgSchG entsprechend dem Thüringer Landesrecht im Thüringer Staatsanzeiger und im Bundesanzeiger veröffentlicht. Dass diese öffentliche Bekanntmachung fehlerhaft sein könnte, trägt auch die Klägerin nicht vor.

Es kann dahin stehen, ob die neben der öffentlichen Bekanntmachung in $\S 6$ Abs. 1 Satz 1 KultgSchG vorgesehene Mitteilung der Eintragung an die Beteiligten als Bekanntmachung eines Verwaltungsaktes im Sinne des § 41 ThürVwVfG einzuordnen ist, die für das Wirksamwerden der Eintragung gegenüber der Klägerin erforderlich wäre. Dagegen spricht schon die Verwendung des Wortes "mitzuteilen", da die damit verbundene Übermittlung einer Information im Verhältnis zur Bekanntgabe ein "Weniger" ist. Dies rechtfertigt die Annahme, dass eine fehlerhafte Mitteilung im Sinne des $\S 6$ Abs. 1 KultgSchG allenfalls für den Beginn des Laufs von Rechtsmittelfristen, nicht jedoch für die Wirksamkeit der Bekanntgabe als solche relevant sein kann. Im Übrigen wäre auch dann von einer wirksamen Bekanntgabe der Eintragung auszugehen, wenn die nach § 6 KultgSchG erforderliche Mitteilung an den beteiligten Eigentümer als Bekanntgabe i. S. d. § 41 ThürVwVfG anzusehen wäre. Auch in diesem Fall wäre die von der Klägerin behauptete fehlerhafte Adressierung des Schreibens vom 16. Dezember 1998 unbeachtlich. Der Verwaltungsakte ist nämlich zu entnehmen, dass R4, der Alleingesellschafter der Klägerin, im Jahre 2003 wegen der Erteilung einer Ausfuhrgenehmigung nach $\S 1$ Abs. 4 KultgSchG bei dem Beklagten vorgesprochen hat. Spätestens ab diesem Zeitpunkt hatte er in der Klägerin zurechenbarer Weise Kenntnis von der Eintragung und ein etwaiger Bekanntmachungsfehler wäre in entsprechender Anwendung des § 9 Thüringer Verwaltungszustellungs- und Vollstreckungsgesetzes - ThürVwZVG - geheilt. 
Überdies spricht viel dafür, dass Herr T zum Empfang der Mitteilung über die Eintragung berechtigt war. Er hat im Namen der Klägerin das an die Familie R gerichtete Schreiben des Beklagten vom 15. September 1998 beantwortet und sich für empfangsberechtigt erklärt. Da er nach Aktenlage auch in anderen Fällen für die Familie R aufgetreten ist, wie sich z. B. aus einem in der Verwaltungsakte befindlichen Protokoll über ein Gespräch mit der Stadt Gera ergibt, gab es aus der Sicht des Beklagten keinen Zweifel daran, dass HerrT für die Klägerin handeln durfte. Sogar dann, wenn er tatsächlich keine Empfangsbevollmächtigung gehabt haben sollte, wäre von einer solchen zumindest nach den Grundsätzen der Anscheins- und Duldungsvollmacht auszugehen.

b. Der Vortrag der Klägerin, das Kulturgutschutzgesetz sei nach Maßgabe des § 18 KultgSchG im November 1997 nicht anwendbar gewesen, vermag ebenfalls keine ernstlichen Zweifel an der Richtigkeit des erstinstanzlichen Urteils zu begründen. Nach § 18 KultgSchG findet das Kulturgutschutzgesetz auf das im öffentlichen Eigentum befindliche nationale wertvolle Kulturgut keine Anwendung, soweit zu dessen Veräußerung nur oberste Bundes- oder Landesbehörden befugt sind oder nach besonderen gesetzlichen Vorschriften die Genehmigung einer aufsichtführenden Stelle der öffentlichen Verwaltung erforderlich ist. Diese Bestimmung ist bezogen auf die „Geraer Silbermöbel“ nicht einschlägig, da diese im maßgeblichen Zeitpunkt der Eintragung im Jahre 1998 in Privateigentum von R2 standen. Es trifft zwar zu, dass die R2 bei Einleitung des Eintragungsverfahrens im November 1997 noch nicht Eigentümerin der "Geraer Silbermöbel" war. Das Eigentum ging erst mit Eintritt der Bestandskraft des Bescheides des Thüringer Landesamtes zur Regelung offener Vermögensfragen - LaRoV - vom 16. Januar 1998 auf die Prinzessin über (vgl. § 12 der gütlichen Einigung vom 21. Oktober 1997). Es ist jedoch kein Grund ersichtlich, warum es dem Beklagten deshalb rechtlich hätte verwehrt sein sollen, schon vor dem absehbaren Eigentumsübergang ein Eintragungsverfahren nach dem Kulturgutschutzgesetz einzuleiten. Zwischen den Beteiligten war der Rückgabeanspruch der R2 nach § 5 AusglLeistG unstreitig, und aus diesem Grund hatte die Stadt Gera auch im Oktober 1997 die gütliche Einigung abgeschlossen. Es stand also im November 1997 schon fest, dass die R2 und nicht die Stadt Gera - oder ein anderer Träger öffentlicher Verwaltung - Eigentümerin werden würde. Selbst wenn man mit der Klägerin davon ausgehen wollte, dass die Einleitung des Eintragungsverfahrens im November 1997 noch nicht zulässig gewesen sein sollte, würden sich daraus keine Gründe ergeben, die einer Eintragung der "Geraer Silbermöbel“ im Oktober 1998 entgegen gestanden hätten.

Schließlich hätte auch auf der Grundlage der - nicht zutreffenden - Auffassung der Klägerin das Eintragungsverfahren spätestens bei Eintritt der Bestandskraft des die Einigung bestätigenden Bescheides des LaRoV Anfang 1998 eingeleitet werden dürfen.

c. Ernstliche Zweifel an der Richtigkeit des erstinstanzlichen Urteils ergeben sich entgegen der Auffassung der Klägerin auch nicht daraus, dass die Geraer Silbermöbel jedenfalls spätestens 1938 in das in Anwendung der "Verordnung über die Ausfuhr von Kunstwerken" vom 11. Dezember 1919 (RGBI. I S. 1961) erstellte Verzeichnis eingetragen waren. Wie das Verwaltungsgericht zutreffend ausgeführt hat, ist Folge dieser Eintragung, dass die Ausfuhr derartiger Kunstwerke auch schon genehmigungspflichtig ist, bevor eine Entscheidung darüber, ob eine Eintragung in das "Verzeichnis national wertvollen Kulturgutes" (§ 1 Abs. 1 KultgSchG) vorgenommen wird oder nicht, getroffen ist (§ $22 \mathrm{Abs} .3$ KultgSchG). Diese Bestimmung war zur „Überbrückung eines Vakuums" erforderlich, da die Verordnung vom 11. Dezember 1919 nach § 22 Abs. 1 KultgSchG außer Kraft gesetzt war (vgl. BT-Drs. 2/76, S. 13). In $\S 22$ Abs. 3 KultgSchG ist kein neben dem Neueintragungsverfahren stehendes Übernahmeverfahren, das anderen Regeln folgt, angeordnet worden. Durch die Verwendung der Worte "in die nach diesem Gesetz auszustellenden Verzeichnisse" wird vielmehr zum Ausdruck gebracht, dass bei der Frage, ob ein Kunstwerk, das bereits unter dem Schutz der Verordnung vom 11. Dezember 1919 stand, eingetragen wird, ebenfalls die Eintragungsvoraussetzungen des $\S 1$ KultgSch $\mathrm{zu}$ prüfen sind. Die Verwendung des Wortes "Übernahme" verdeutlicht nur die Verpflichtung der Behörde, schon von Amts wegen die in die alten Verzeichnisse eingetragenen Kunstwerke besonders für eine Eintragung in das Verzeichnis nach § 1 Abs. 1 KultgSchG in den Blick zu nehmen. Die Auffassung der Klägerin, dass bei der Eintragung derartiger Kunstwerke nach $\S 1$ Abs. 1 KultgSchG zu prüfen sei, ob die Voraussetzungen der Verordnung vom 11. Dezember 1919 noch vorliegen, ist angesichts dieses eindeutigen Wortlauts nicht haltbar.

d. Das Urteil des Verwaltungsgerichts begegnet auch keinen ernstlichen Zweifeln, soweit es den geltend gemachten Löschungsanspruch nach $\S 7$ KultgSch G mit der Begründung verneint hat, dass eine wesentliche Veränderung der Umstände nicht ersichtlich sei. Wie bereits ausgeführt, müssen für die Eintragung eines Kunstwerks, das bereits nach Maßgabe der Verordnung vom 11. Dezember 1919 unter Schutz gestellt war, in das "Verzeichnis national wertvollen Kulturgutes" die Voraussetzungen des § 1 Abs. 1 KultgSchG vorliegen. Aus diesem Grund ist es nur folgerichtig, bei der Prüfung der Frage, ob sich die Umstände wesentlich verändert haben, vergleichend an die Umstände im Zeitpunkt der Eintragung anzuknüpfen. Die "Geraer Silbermöbel“ wurden 1998 nach Maßgabe des § 1 Abs. 1 KultgSchG in das "Verzeichnis wertvollen Kulturgutes" eingetragen. Sowohl der Verlust des Wandspiegels im Jahre 1945 als auch die Eintragung des sog. „Welfensilbers“ im Jahre 1987, die durch das Urteil des Bundesverwaltungsgerichts vom 27. Mai $1993-7$ C 33/92 - (BVerwGE 92, 288-294) bestandskräftig wurde, sind Umstände, die im Zeitpunkt der Eintragung schon eingetreten und dem Beklagten auch bekannt waren. Deshalb hätten auf diese Umstände bezogene Einwände bereits in einem gegen die Eintragung gerichteten Verfahren erhoben werden müssen. Die Eintragung ist jedoch bestandskräftig und aus den bereits genannten Gründen auch wirksam. In einem Löschungsverfahren können solche Einwände, die bereits gegen die Eintragung hätten erhoben werden können, nicht mehr geltend gemacht 
werden. Dass sich die Umstände seit der Eintragung der Geraer Silbermöbel im Jahre 1998 wesentlich verändert haben, behauptet auch die Klägerin nicht.

2.) Die Rechtssache weist auch keine besonderen tatsächlichen oder rechtlichen Schwierigkeiten im Sinne des § 124 Abs. 2 Nr. 2 VwGO auf. Derartige Schwierigkeiten liegen nur dann vor, wenn die Angriffe des Rechtsmittelführers gegen die erstinstanzliche Entscheidung Fragen von solcher Schwierigkeit aufwerfen, die nicht ohne weiteres im Zulassungsverfahren, sondern erst im eigentlichen Rechtsmittelverfahren geklärt werden können (vgl. Senatsbeschluss vom 12.12.1997 - 1 ZEO 1118/97 -). Derartige Fragen wirft die Klägerin jedoch nicht auf.

a. Die Frage, ob bei der Eintragung eines Kunstwerks in das „Verzeichnis national wertvollen Kulturgutes" zwischen einer Neueintragung und einer Übernahme zu unterscheiden ist, lässt sich aus den unter 1.) genannten Gründen auch ohne Durchführung eines Berufungsverfahrens in der Weise beantworten, dass es ein gesondertes Übernahmeverfahren nicht gibt. Auch für die Kunstwerke, die bereits unter dem Schutz der Verordnung vom 11. Dezember 1919 standen, ist vor einer Eintragung zu prüfen, ob die Voraussetzungen des § 1 Abs. 1 KultgSchG vorliegen.

b. Auch der Vortrag der Klägerin, der nach Maßgabe des $\S 2$ Abs. 2 KultgSchG vor der Eintragung zu beteiligende Sachverständigenausschuss sei fehlerhaft besetzt gewesen, rechtfertigt weder die Zulassung der Berufung nach § 124 Abs. 2 Nr. 2 VwGO noch aus anderen Gründen. Wie bereits ausgeführt, ist die Eintragung selbst wirksam und bestandskräftig. Gegenstand dieses Verfahrens ist der geltend gemachte Löschungsanspruch. In diesem Verfahren können Gründe, die sich auf eine eventuelle Rechtswidrigkeit der Eintragung selbst beziehen, nicht geltend gemacht werden. Ergänzend kommt hinzu, dass die Beteiligung des Sachverständigenausschusses nach $\S 2$ Abs. 2 KultgSchG in einem Löschungsverfahren nach $\S 7$ KultgSchG nicht vorgesehen ist. Daran ändert auch der Umstand, dass der Beklagte dies anders gesehen und vorsorglich eine Beteiligung durchgeführt hat, nichts.

Ebenso wenig vermag die von der Klägerin zitierte Kommentierung von Bernsdorff/Kleine-Tebbe zu $§ 22$ KultgSchG eine andere Einschätzung zu rechtfertigen, da sich diese auf Fälle der erstmaligen Eintragung von Kunstwerken, die nach der Verordnung vom 11. Dezember 1919 unter Schutz standen, und nicht auf die Löschung nach $\S 7$ KultgSchG bezieht.

3.) Schließlich ist die Berufung auch nicht wegen der von der Klägerin geltend gemachten grundsätzlichen Bedeutung der Rechtssache (vgl. § 124 Abs. 2 Nr. 3 VwGO) zuzulassen. Grundsätzliche Bedeutung in diesem Sinne hat eine Sache dann, wenn sie eine klärungsbedürftige Frage des materiellen oder formellen Rechts aufwirft und zu erwarten ist, dass die Entscheidung im zweitinstanzlichen Verfahren dazu dienen kann, die Rechtseinheit in ihrem Bestand zu erhalten oder die Entwicklung des Rechts zu fördern (vgl. etwa BVerwG, Beschluss vom 2. Oktober 1961 -BVerwG VIII B 78.61 -, BVerwGE 13, 90/91;
Senatsbeschluss vom 6. September 1995 - 1 ZO 388/94 -, BRS 57 Nr. 173). Ausschlaggebend ist demnach nicht das Interesse des Einzelnen an der Entscheidung, sondern das abstrakte Interesse der Gesamtheit an der einheitlichen Entwicklung des Rechts. Die Zulassung der Berufung wegen grundsätzlicher Bedeutung der Rechtssache ist nur dann gerechtfertigt, wenn zu erwarten ist, dass in der Berufungsentscheidung eine klärungsbedürftige Frage mit Verbindlichkeit über den Einzelfall hinaus in verallgemeinerungsfähiger Form beantwortet werden kann (vgl. ThürOVG, Beschluss vom 17.6.1997 - 3 ZKO 217/97 -, NVwZ 1998, 194).

Die Klägerin hält für grundsätzlich klärungsbedürftig, ob das auf der Grundlage des § 5 AusglLeistG erlangte Eigentum an einem Gegenstand durch eine Eintragung in das „Verzeichnis national wertvollen Kultgutes" beschränkt werden darf. Diese Frage ist nicht klärungsbedürftig, weil sie sich auch ohne Durchführung eines Berufungsverfahrens beantworten lässt. Durch die Rückgabe einer beweglichen Sache nach Maßgabe des § 5 AusglLeistG erhält der Berechtigte Eigentum im Sinne des Art. 14 GG, dessen Inhalt und Schranken gemäß Art. 14 Abs. 1 Satz 2 GG durch die Gesetze bestimmt wird. Es ist bereits höchstrichterlich geklärt, dass das Kulturgutschutzgesetz mit seinen sich daraus ergebenden Einschränkungen für die Veräußerbarkeit eine verfassungsgemäße Inhalts- und Schrankenbestimmung im Sinne des Art. 14 Abs. 1 Satz 2 GG ist (vgl. BVerwG; Urteil vom 27. Mai 1993 - BVerwG 7 C 33.93 - BVerwGE 92, 288/291).

\section{III.}

Die Kostenentscheidung folgt aus $\S 154$ Abs. 2 VwGO. Die Streitwertfestsetzung beruht auf $\S \S 63$ Abs. 2, 47 und 52 Abs. 1 Gerichtskostengesetz - GKG - in der seit dem 1. Juli 2004 geltenden Fassung. Das für die Bestimmung des Streitwerts nach $\S 52$ Abs. 1 GKG maßgebliche Interesse an der Aufhebung an der Beseitigungsanordnung bemisst der Senat wie die Vorinstanz mit 2.000.000,00€.

Der Beschluss ist unanfechtbar (§ 152 Abs. 1 VwGO, § 68 Abs. 1 Satz 5 i. V. m. § 66 Abs. 3 Satz 3 GKG). 